\title{
'Mis Hijos se Quedan Allá': Estrategias Cotidianas de Mujeres Urbanas que Trabajan en el Turismo de Aventura en 'Rio-Tlan”, México
}

\author{
'My Kids are Over There': Everyday Strategies of Urban Women \\ Working in Adventure Tourism in 'Rio-Tlan', Mexico
}

Isis Arlene Díaz-Carrión

Universidad Autónoma de Baja California

diaz.isis@uabc.edu.mx

\section{Resumen}

A través de los estudios de la cotidianidad, la Geografía de género ha podido visibilizar las diferencias espacio-temporales experimentadas por las mujeres como resultado del trabajo reproductivo y productivo. En este sentido se revisan las estrategias realizadas por mujeres urbanas para cubrir con los cuidados de los hijos y de la casa cuando el lugar de trabajo, vinculado al turismo de aventura, se ubica en el medio rural.

Palabras clave: vida cotidiana; género; relación urbano-rural.

\section{Abstract}

By means of everyday life studies, Gender geography can make visible the time-space differences experienced by women as a consequence of reproduction and productive work. Special attention is given to daily life strategies performed by urban women in order to solve childcare and housework duties when their workplace, related to adventure tourism, is located in the countryside.

Keywords: Everyday life; Gender; Urban-Rural relations. 


\section{Introducción}

El análisis de las situaciones y trayectorias cotidianas han sido preciado objeto de estudio por parte de diversas disciplinas, destacando entre éstas los estudios sociológicos que han dedicado múltiples trabajos. ${ }^{1}$ Alejada de los grandes acontecimientos, los estudios de la vida cotidiana se interesan en abordar las vivencias a través de las cuales se construyen diferentes discursos que terminan convirtiéndose en referentes sociales de los individuos (POLLIO et al., 2006), pero también por permitir incorporar, articular y confrontar distintas dimensiones de la vida social (LINDÓN, 2002).

Por ello, la Geografía de género construye alrededor de la vida cotidiana una serie de líneas de estudio encaminadas a dar fe de los efectos del género - y otras formas de exclusión social - en la construcción y usos del tiempo y espacio. En este sentido, diversos estudios han visibilizado la construcción de una cotidianidad de las mujeres cercana a la casa como consecuencia de la realización del trabajo reproductivo, ${ }^{2}$ generándose un efecto de anclaje físico de éstas (LINDÓN, 2006) con efectos sobre su movilidad (HANSON \& HANSON, 1981).

Buscando arrojar luz sobre las restricciones espacio-temporales de las mujeres mexicanas que no han podido - o deseado- encontrar cabida en el mercado laboral local, se estudia la situación de RioTlan, una comunidad rural que atrae a diario mano de obra calificada desde las ciudades circunvecinas. Ante un trabajo productivo que tiene lugar fuera de las poblaciones urbanas de residencia, se propone explorar los acuerdos de las mujeres para resolver las necesidades reproductivas de sus grupos domésticos, prestando atención a las características intrínsecas de dichos grupos a fin de identificar posibles variaciones, prestando atención a la figura de anclajes que pudiera generar el trabajo reproductivo.

También de interés es el monitoreo de estrategias utilizadas para compatibilizar el trabajo reproductivo y el productivo; con este objetivo se revisan los acuerdos planteados por mujeres cuyos grupos domésticos son unipersonales o nuclear-estricto, buscando identificar si las estrategias ante el cuidado de los hijos tienen precedente entre mujeres con ciclos de vida previos.

\section{Espacios de vida y el enfoque de género}

Para la Geografía humana el estudio de la vida cotidiana ha sido una de las consecuencias de abrazar un desafío postmoderno y le ha permitido vincularse estrechamente con la teoría social (LINDÓN y HIERNAUX, 2006), este giro comienza como consecuencia de una serie de artículos publicados en 1980: “(...) basados en enfoques ya existentes para la interpretación del paisaje que llevaron a nuevas alianzas con los estudios poscoloniales y feministas" (JACKSON, 1999, p. 42) los cuales rápidamente tuvieron un efecto reformador - el redescubrimiento de la dimensión cultural en la Geografía (LINDÓN y HIERNAUX, 2006) - en el conjunto de la disciplina.

La Geografía humana ha estudiado la cotidianidad a través de los desplazamientos, las prácticas que permanecen en un lugar, los escenarios de comportamiento y los patrones o rutinas espaciales (LINDÓN, 2006). Atendiendo la espacialidad, dan cuenta de las múltiples articulaciones existentes entre las experiencias interiores y los fenómenos exteriores. Pero, si bien la vida cotidiana puede remitirnos a un espacio sinónimo de 'libertad' también nos muestra el lugar definido a través de prácticas de anclaje a un espacio limitado (LAEGRAN, 2008; SCHWANEN et al., 2008). Es por ello que la vida cotidiana ha sido uno de los principales objetos de análisis de otra de las, según considera Jackson (op. cit.), 'Nuevas Geografías': la Geografía del Género o feminista.

La Geografía del Género hurga en la cotidianidad con el ánimo de identificar las diversas estructuras que limitan - abierta y soterradamente - las trayectorias espacio-temporales de las mujeres (ROSE, 1993). Para la Geografía del Género, la vida cotidiana - como arena donde se sostienen y modifican las relaciones de poder- se equipara con la vida social y por lo tanto no es indiferente al género sino que tradicionalmente ha circunscrito a las mujeres con el espacio-tiempo reproductivo (SABATÉ et al., 1995).

Dentro de los estudios que recurren a la cotidianidad y género, aquellos de la percepción y los usos del espacio constituyen una de las líneas más exploradas; uno de los primeros trabajos que indaga sobre la participación social y económica en la vida local es el de Everitt quien de forma un tanto involuntaria registra pautas de interacción espacial diferenciados entre mujeres y hombres que trabajan fuera de casa (ibídem). Dentro de los primeros trabajos que asumen abiertamente la importancia del género en el día a día destacan los trabajos de Hanson en Uppsala, a través de esta serie de estudios se visibilizan el papel del espacio en la construcción social de los roles de género, así como las limitaciones impuestas por la geografía a la vida laboral de las mujeres (HANSON, 1980; HANSON \& HANSON 1980 y 1981). Posteriormente, en esta línea de investigación destacan trabajos que demuestran el papel de las diferencias culturales al momento de definir los espacios mentales (KATZ \& MONK, 1993; NELSON, 2006), así como las diferencias 
generacionales o, más recientemente, las posibles vinculaciones entre el género y la movilidad sostenible (HANSON, 2010); dando cuenta de la riqueza de análisis que tienen como punto de partida los usos del espacio y género.

\section{Tiempos y espacios que sostienen la vida de las mujeres}

Sin embargo como anotan Sabaté et al. (op. cit.) el entorno cotidiano también ha permitido a la Geografía del Género la exploración de experiencias, vivencias, sentimientos y valores de las mujeres - y los hombres con sus entornos; los cuales se convierten, a su vez, en una base recurrente en la construcción de sus respectivas identidades.

De tal suerte que en la búsqueda de significados, la vida cotidiana ha sido también revisada como consecuencia del trabajo y los usos espacio-temporales que lo sustentan (LINDÓN, 2002; RILEY, 2009; QUINN, 2010). Son los espacios de vida, que van entretejiendo las dimensiones de la vida social con diversas estrategias desarrolladas - y en buena medida también sostenidas - por las mujeres a quienes tradicionalmente se les ha asignado la responsabilidad de realización del trabajo reproductivo, pero que a través de los estudios de la vida cotidiana van apareciendo las aportaciones productivas $\mathrm{y}$ comunitarias que han podido permanecer invisibles por considerarse exclusivamente roles propios de los varones. Indudablemente, estos espacios en parte se materializan a través de los estudios de movilidad, pero igualmente permiten una revisión más a fondo de las experiencias que resultan ya no sólo de las trayectorias, o de la unión de los diversos espacios sino también de cómo las dinámicas cotidianas son 'sentidas' y 'vividas' por las personas involucradas.

El estudio de la vida cotidiana ha permitido a Townsend (et al., 1994) estudiar las estrategias locales en los procesos de colonización, así como el peso de las identidades de género sostenidas a través de la división del trabajo; una línea que continúa vigente, permitiéndose dar seguimiento a nuevas dinámicas internacionales de expulsión de la población (migración) en territorios colonizados apenas unas décadas atrás (RADEL et al., 2010); procesos - si no de descolonización, si al menos de expulsión - que terminan generando en los grupos domésticos espacialidades discontinuas tanto en la escala macro como en la micro, siendo éstas últimas experimentadas directamente por las mujeres 'que se quedan' y viven su cotidianidad con largas jornadas de trabajo -reproductivo, productivo y comunitario.
Asimismo la Geografía del Género ha explorado la vida cotidiana como expresión de empoderamiento de las mujeres a través de dinámicas políticas (MATTINGLY y HANSEN; 2006; NELSON, 2004) o de reivindicación de derechos (AYLLÓN, 2007; BOSCO et al., 2011); donde los tiempos y espacios de vida son recurridos para confrontar la identidad tradicional.

A través de la revisión del trabajo, la vida cotidiana de las mujeres ha permitido explorar tanto el cuestionamiento de las ideas locales construidas entorno a lo femenino y lo indígena (NELSON, 2006) o el trabajo productivo (CHAMBERS \& HENSHALLMOMSEN, 2007); trabajos que apuntan a las vivencias personales para desentrañar las identidades y la forma en que éstas interactúan y pueden ser cambiadas modificando las trayectorias espacial y temporal.

Otro eje analítico define a la vida cotidiana como soporte de las identidades transgresoras contextualizándolas en el marco de la división del trabajo según género, las normas sociales y de otros acuerdos locales enfrentados a macro procesos de diversa índole - particularmente la económica(NELSON, 2006; McEVOY et al., 2012); convirtiendo a la cotidianidad en un medio para dar cuenta de todas las estrategias de vida social y de desafíos que pueden parecer mínimos pero cargados de significado para, en estos casos, las mujeres que abren y conquistan nuevas prácticas espaciales.

\section{Zona de Estudio}

Rio-Tlan es el nombre imaginario de una comunidad real ubicada en el medio rural del sur de México; hasta el imaginado Rio-Tlan y sus ciudades vecinas se han extrapolado dinámicas de diversa índole a fin de reconstruir las trayectorias espaciotemporales de mujeres urbanas que constituyen una mano de obra calificada para el turismo de aventura de Rio-Tlan. ${ }^{3}$

Rio-Tlan es entonces una cabecera municipal ubicada a unos $40 \mathrm{kms}$. de la capital de alguno de los sureños Estados del país. Hasta mediados de los 1990 Rio-Tlan era una comunidad con una marcada actividad agrícola, pero la crisis del agro y la llegada de 'urbanitas' que practicaban 'rafting' han ido cambiando el uso de las tierras y donde antes se cultivaba cacahuate ahora hay campamentos de turismo de aventura.

La elección de Rio-Tlan como caso de estudio, y no las ciudades que le proveen de mano de obra, se debe al interés de estudiar la cotidianidad de mujeres que trabajan fuera de casa y realizan desplazamientos diarios mayores a los de sus parejas; en la muestra 
elegida, ninguna de las parejas o esposos presentan desplazamientos diarios mayores a los de las mujeres que trabajan en el turismo de aventura en Rio-Tlan.

\section{Anotaciones metodológicas}

Esta investigación recurre a la entrevista en profundidad semi-estructurada, la observación indirecta y el análisis de documentos visuales como principales herramientas. La muestra de estudio de esta investigación se refiere precisamente a mujeres que residiendo en el medio urbano trabajan en el turismo de aventura en Rio-Tlan; se trata de 20 mujeres entre las edades de 24 y 43 años ${ }^{4}$, los grupos domésticos de la muestra de estudio son unipersonales y de tipo nuclear en diversas de sus tres sub-modalidades estricto (pareja sin hijos), conyugal (pareja con hijos) y monoparental (madre o padre con hijos). La muestra se compone de mujeres con estudios universitarios principalmente de licenciatura, aunque en algunos casos se posee también el grado de maestría - en todos los casos se trata de empleadas de tiempo completo en puestos ubicados en el nivel medio y gerencial de los diversos campamentos; se puede hablar, en general de personas de clase media-alta y alta-baja.

Todas las mujeres entrevistadas se desplazan de lunes a viernes de 8:00 a 18:00 horas y sábados de 8:00 a 15:00 hrs, desde la capital del Estado y ciudades vecinas hasta los campamentos de turismo de aventura de Rio-Tlan; en esta franja horaria está contemplado su horario de trabajo, el tiempo dedicado a la comida y el desplazamiento; no obstante, hay que señalar que es básicamente orientativo y que en ocasiones los requerimientos de trabajo (p. ej. cuando hay grupos o eventos especiales) requieran de horas extras y en el caso de algunos puestos gerenciales una disposición aún mayor.

\section{La organización de la vida cotidiana: caso empírico}

En la mayoría de los estudios de percepción del espacio, suele prevalecer la figura de una mujer cuya vida cotidiana se construye físicamente si no alrededor, sí cerca del domicilio (HANSON \& PERRY, 1981) visibilizando un efecto de anclaje como consecuencia del trabajo doméstico, de socialización y cuidados (LINDÓN, 2006).

Pero ¿Qué sucede cuándo son las mujeres quienes se desplazan mayores distancias que los hombres? ¿Cómo se resuelve el trabajo reproductivo? Ante la ausencia de la madre ¿Se detona la participación de los hombres en el trabajo reproductivo?

\section{La vida cotidiana de las mujeres sin hijos}

Las mujeres sin hijos pertenecen a dos tipos de grupos domésticos: los unipersonales y los nucleares (de tipo estricto y conyugal); la presencia de mujeres sin hijos -casi todas solteras- que viven solas es pequeña si se compara con el resto, si bien la muestra de estudio no es estadísticamente representativa se quiere dejar constancia de la tendencia a encontrar a las mujeres formando grupos nucleares. ${ }^{5}$

Las mujeres sin hijos en hogares unipersonales y nucleares resuelven sus requerimientos de trabajo doméstico a través de diversas estrategias, pero la mayor carga la asumen ellas mismas:

Algunas cosas como ir al súper pues lo hago yo en la quincena ¿no? lo de limpiar y eso lo hago yo, ya sabes una barridita y eso así muy por encimita [risa] y pues mi depa6 es pequeño y trato de ser ordenada así que en unas horas ya queda listo (MICHELLE, 30 años, sin hijos, grupo doméstico unipersonal).

En el caso de las mujeres en hogares nucleares aunque interviene el resto del grupo doméstico es posible apreciar la feminización de ciertas actividades, ${ }^{7}$ pero una mirada más detallada permite identificar como una actividad puede desglosarse a su vez en micro actividades tendientes a feminizarse:

por lo regular quienes cocinamos o
desenvolvemos la comida comprada y
estamos pendientes de que la mesa esté
servida, y eso y estamos al pendiente de los
niños somos las mujeres; los hombres están
que en el futbol o el béisbol, ayudan al
recoger la mesa y a veces a lavar y secar
los trastes (MARTINA, 31 años, hijo
pequeño, grupo doméstico nuclear
conyugal).

Todas las mujeres consideran que los hombres también participan en la realización de trabajo doméstico; en algunos casos esta realización es catalogada de ayuda, entendiendo que la principal responsable es la mujer; pero entre las mujeres más jóvenes la participación del esposo, la pareja o los hermanos en la realización del trabajo doméstico es entendida como una corresponsabilidad: “(...) en mi casa no hay eso de que porque es hombre no hace nada, cada quien se encarga de su cuarto, de su ropa y las cosas comunes nos las dividimos" (MÓNICA, 25 
años, sin hijos, grupo doméstico nuclear conyugal).

No obstante, incluso en estos casos es una mujer la de mayor edad - quien suele mantener la principal responsabilidad de organización y/o realización del trabajo doméstico: "Las compras más importantes, el control de lo que ya se ha pagado y eso de que llegues, abras tu casa y esté limpia o el refril y haya comida pues sí, se encarga mi mamá" (MAGALI, 24 años, sin hijos, grupo doméstico nuclear conyugal).

La figura de la madre aparece también como un apoyo importante en el caso de las hijas, vivan solas: "mi mamá cuando va al mercado me habla por si quiero algo, como fruta fresca o carne fresca y ya ella me la compra y paso por ella a su casa, o si va a pagar que la luz o el teléfono y si quiero que pague mi recibo" (Marina, 33 años, sin hijos, grupo doméstico unipersonal), o con pareja:

(...)sí, la verdad es que mi mamá es un súper apoyo, Roberto [su pareja] y yo cenamos todas las noches con ella o sea que yo por comidas al día en la semana pues... solamente me preocupo del desayuno... porque yo como aquí en el campamento, Roberto come con su mamá o en la calle y el desayuno pues ahí nos la turnamos y cositas así de juguitos, cerealito, yogurcito $y$ sandwichito, eh! (MONTSERRAT, 29 años, grupo doméstico nuclear estricto).

Otra de las estrategias recurridas para la resolución del trabajo doméstico es la contratación de ciertos servicios con el mercado, esta opción se aplica principalmente a las tareas de limpieza: "una vez por semana va una señora a limpiar la casa y a planchar la ropa, el resto nos lo dividimos entre mi pareja y yo" (MARÍA, 33 años, grupo doméstico nuclear estricto); de alimentación: "si tengo flojera de cocinar compro comida o si tengo ganas pues quedo con alguien a comer fuera" (MICHELLE, op. cit.); y algunos servicios:

(...) pues como ando todo el día hasta acá aprovecho todo lo que sean pagos por Internet o por medio del banco que si luz, teléfono, la tarjeta, el cable o eso se encarga el banco de que se paguen, pagas una comisión por eso pero pues de otra forma me es imposible" (MONTSERRAT, op. cit.).

La contratación de servicios domésticos con el mercado es una importante estrategia a la que se recurre sobre todo si se tiene poder adquisitivo importante o si se carece de una red de apoyo familiar o extra-familiar, como sucede en el caso de Mar (39 años, sin hijos, grupo doméstico unipersonal) cuya red familiar y extra-familiar se ubica en otra ciudad distante: "Yo aquí llegué y no conocía a nadie, pues... yo no tengo eso de que a veces veo en mis amigas que la mamá les apoya un montón y pues lo de las compras, las reparaciones, la casa y eso soy yo sola quien lo resuelvo ¿No?”.

Para la mayoría de las mujeres que trabajan en RioTlan a pesar de formar grupos domésticos unipersonales o nucleares la familia ampliada continúa siendo un soporte importante al momento de ir conciliando la vida personal y laboral; las redes familiares (madres, hermanas o primas) son una fuente de recurrencia tanto para las cosas ordinarias como para las extraordinarias:

(...) hace como un año tuvieron que operarme por apendicitis y si, pues en el hospital se turnaban entre mi mamá, mi hermana y mi esposo, bueno novio, pareja, bueno eso... ya que me dieron de alta no me fui a mi casa sino que me quedé, o bueno nos quedamos porque pues él [pareja] también se fue para allá... en casa de mis papás estuvimos hasta que ya me dieron de alta (MIREYA, 26 años, grupo doméstico nuclear estricto).

Estas relaciones de apoyo se dan en ambos sentidos pues las mujeres declararon estar pendientes de lo que sucedía con el resto de su familia, una relación que aún resulta fuertemente sostenida a partir de los esfuerzos de las mujeres: "yo hablo con mi mamá pues... casi a diario" (MARÍA, op. cit.), y que se buscan mantener incluso a la distancia:

(...) mis papás están divorciados y ninguno de los dos vive en la ciudad donde vivo yo, entonces pues estoy en contacto con ellos por email, o chateamos o por el teléfono y cada que puedo me escapo a verlos o vienen ellos, pero sí... es con mi mamá, luego con mi hermano, y luego con mi papá, así en ese orden es que estoy pendiente de ellos ¿No? (MAR, op. cit.). 
'Mis Hijos se Quedan Allá': Estrategias Cotidianas de Mujeres Urbanas que Trabajan en el Turismo de Aventura en 'Rio-Tlan', México

\section{Cotidianidad de mujeres con hijos pequeños y adolescentes}

Independientemente de si la mujer conforma un grupo doméstico nuclear-conyugal o nuclearmonoparental son ellas las principales responsables del trabajo doméstico y la participación del esposo o la pareja - en el caso de los grupos nucleares-conyugalesse mantiene como una participación puntual, que bien sirve de enlace entre los espacios cotidianos de socialización y cuidados: "mi esposo pasa por mis hijos a la casa de mi mamá cuando (...), cuando por ejemplo a mí se me hace tarde, él sale de su trabajo y los recoge, sino se va derecho a la casa" (MANUELA, 35 años, hijos adolescentes, grupo doméstico nuclear conyugal) o ante situaciones extraordinarias: "si... haz de cuenta que si por ejemplo a la niña le pasara algo en la escuela... primero tratarían de localizar a mi mamá, luego a mí y ya si no hablarían con mi esposo" (MIRNA, 37 años, hijos adolescentes, grupo doméstico nuclear conyugal). ${ }^{9}$

$\mathrm{La}$ presencia de la abuela es un elemento que determina en buena medida el resto de las estrategias recurridas por las mujeres para suplir su ausencia mientras trabajan en Rio-Tlan; lo anterior se mantiene incluso cuando el nivel económico puede permitir la adquisición en el mercado de servicios de cuidados y/o de trabajo doméstico. Para las mujeres urbanas que trabajan en Rio-Tlan, el contar con una abuela pendiente del cuidado de los hijos

especialmente si son pequeños- da lugar a una serie de estrategias cotidianas donde el espacio fragmentado se va enlazando en buena medida a partir de la presencia de la abuela.

Una estrategia recurrida es precisamente la de convertir la casa de la abuela en el espacio donde permanecen los hijos una vez que han salido de la escuela y hasta que, en la mayoría de los casos, la madre pasa por ellos; en este caso si bien el grupo doméstico es nuclear presenta funciones espaciales propias de uno de tipo ampliado, pero donde se destaca una discontinuidad espacial: "Mis hijos es como si tuvieran dos casas, todo lo tienen doble, que si en nuestra casa o en la de la abuelita... o si no tienen que andar cargando con libros o juguetes de una casa a la otra" (MANUELA, op. cit.). En estas situaciones la casa del grupo doméstico nuclear cumple, sobre todo, entre semanas con la función de dormitorio mientras que el resto de la vida cotidiana de los hijos tiene lugar en otros espacios públicos -la escuela, la calle, p. ej.- y en la casa de la abuela.

Esta fragmentación del espacio puede llevarse al máximo en algunas circunstancias, sobre todo cuando la casa del grupo nuclear se ubica en sectores de la ciudad con carencias de servicios:

(...) durante un tiempo vivimos en una colonia en las afueras... si el niño se me enfermaba nos quedábamos todos en casa de mi mamá, yo me sentía más tranquila porque sabía que si se me ponía más malo estábamos más cerca del doctor, del hospital, de las farmacias (MACARENA, 43 años, hijos adolescentes, grupo doméstico monoparental).

El papel de las abuelas en el cuidado de los nietos si bien disminuye conforme van creciendo suele mantenerse para cubrir las actividades de alimentación y cuidados: "ya que mis hijos han ido creciendo mi mamá ha dejado de estar tan pendientes de ellos... ya por ejemplo no tiene que ir por mi hijo más pequeño al salir de la escuela, ya se va sólo... pero sí, siguen yendo a comer a su casa" (id.).

La presencia de la abuela compartiendo el espacio doméstico con los nietos ha sido una garantía de tranquilidad: “....yo estoy pendiente de ellos (los hijos) a la hora de la comida llamo a casa de mi mamá para ver cómo están, o si alguno está enfermo pues lo hago más veces al día" (MAYRA, 39 años, hijo pequeño, grupo doméstico ampliado), con el incremento de la inseguridad y la violencia en el Estado de Veracruz, el papel de la abuela el cuidado de los nietos se ha visto reforzado: "Me da miedo por mis hijos, las cosas están muy revueltas y pues que sepa que está mi mamá allá pendiente de ellos me tranquiliza" (MANUELA, op. cit.).

Entre las mujeres entrevistadas es poco frecuente la existencia de una remuneración informal hacia las abuelas que cuidan de los nietos; lo más común es el intercambio de servicios - principalmente de cuidados - en una especie de banco de tiempo, sólo que en este caso el intercambio se realiza entre familiares. Las mujeres que trabajan en Rio-Tlan realizan para los grupos domésticos de las abuelas diversas actividades, pero de manera general se pueden señalar como recurrente la compra en los supermercados: "cada quincena me pongo de acuerdo con mi mamá y en domingo por la tarde vamos al super mercado yo hago mi compra y ella la suya" (MACARENA, op. Cit.) ${ }^{10}$; la realización de gestiones - particularmente las que impliquen utilización de las nuevas tecnologías-: “(...) pues lo de hacer pagos a través de Internet, o lo de retirar dinero del cajero, sí en eso les echo una mano" (MAGDALENA, 33 años, hijas pequeñas, grupo doméstico nuclear conyugal); si bien en este tipo de intercambios es frecuente encontrar la participación de los nietos, éste suele presentarse a requerimiento de las 
madres de éstos: “(...) a mi hijo siempre que si hay que pagar el cable, o el teléfono o eso son cosas que le pido haga por su abuela" (MACARENA, op. cit.).

Otra de las actividades que suelen entrar en este intercambio de trabajo están muy relacionadas con los servicios de cuidado; en México es todavía bastante generalizado el papel de los hijos - más específicamente de las hijas - en el cuidado de los padres cuando estos comienzan a hacerse mayores o cuando experimentan alguna enfermedad. Si las abuelas no cuidaran de los nietos ¿Se darían estos intercambios? anteriormente se mencionó la presencia de cuidados de los padres entre las mujeres con grupos domésticos unipersonales; identificándose a esta serie de intercambios como parte de las relaciones familiares, como comenta Mariana (29 años, hijo pequeño, grupo doméstico ampliado): “(...) pues sí, mi mamá me cuido yo la veré cuando sea viejita". No obstante, cuando las abuelas cuidan de los nietos suele registrarse un aumento de los servicios domésticos, de socialización y de cuidados intercambiados: "Siempre he estado al pendiente de mi mamá y de mi papá, pero desde que nació la niña y ella me la cuida pues sí, estás como que más al pendiente de ellos ¿No?" (MARIBEL, 40 años, hija pequeña, grupo doméstico nuclear conyugal).

Ante la ausencia de una persona para supervisar el cuidado de los hijos y/o de la casa o cuando el grupo doméstico puede permitírselo se presenta la contratación de trabajo doméstico en el mercado, contratando principalmente aquellas actividades impostergables. En este sentido cabe señalar la prevalencia de contratación con el Estado o el mercado de una parte importante del trabajo de socialización de los menores: el de la educación; por otro lado, en lo referente a los servicios de cuidados éstos suelen resolverse a través de guarderías bien públicas, bien privadas pudiendo éstas últimas ser colectivas: "Llevo al niño a una guardería que es toda de paga" (MARIANA, op. cit.), o particulares: "yo le pago a una señora para que cuide de las gemelas en la casa, ella las lleva al maternal, a la salida va por ellas y se las lleva a casa, les da de comer, las baña, juega con ellas hasta que llego yo" (MAGDALENA, op. cit.). ${ }^{11}$

Para las actividades de trabajo doméstico postergables - la mayor parte de éstas tienen que ver con el cuidado de la casa - las mujeres buscan resolverlas en su tiempo libre, particularmente durante su día de descanso. La participación de los hijos resulta importante en la mayoría de los grupos domésticos; no obstante, entre más mujeres familiares contratadas - estén dispuestas para la realización del trabajo doméstico menor será la cuota de trabajo de los hijos. ${ }^{12}$

Cuándo contratar trabajo reproductivo con el mercado es una estrategia en función del nivel económico: "antes que ganábamos mucho menos (ella y el esposo) no podíamos pagar por ello" (MIRNA, op. cit.), la ausencia de alguna familiar: "antes de divorciarme vivíamos en el D.F. (Ciudad de México) y allá pues no, la única forma era pagarle a una señora, Doña Caro, para que estuviera pendiente de ellos (los hijos), ella vivía con nosotros, se quedaba entre semana y el fin de semana se iba a su pueblo" (MACARENA, op. cit.). No obstante, persiste la idea de considerar a la familia como la mejor figura de cuidado de los hijos, por lo que la familia puede convertirse también en principal proveedora de trabajo reproductivo retribuido:

\section{(...) cuando mi primer hijo nació intenté llevarlo a la guardería... estaba súper lejos y perdía muchísimo tiempo, luego una vez se enfermó se puso malísimo estuvo varios días en urgencias, el doctor nos dijo que el virus que le había dado era un contagio muy común en las guarderías; fue entonces cuando mi mamá se ofreció a cuidarlo en casa y entonces comencé a pagarle a ella... yo también así pues me sentí más tranquila (MIRNA, op. cit.).}

Finalmente comentar la consideración de corresponsabilidad en la participación de hijos y las hijas en el trabajo reproductivo, corresponsabilidad que, principalmente entre las mujeres más jóvenes llega a incluir al esposo o pareja; si bien lo anterior se presenta indudablemente como una importante apuesta a futuro, es posible también encontrar la permanencia de tareas feminizadas.

\section{Consideraciones Finales}

Los estudios de la vida cotidiana han sido útiles como herramienta para vincular los modos de producción y reproducción de la vida material enriqueciendo las interpretaciones al visibilizar, especialmente en las mujeres pero también en los hombres, las intrincadas relaciones que superan artificiales divisiones de trabajos, espacios y tiempos de vida. La vida cotidiana evidencia un constante fluir entre tiempos y espacios a través de los cuales las mujeres logran unir, a veces precariamente, las responsabilidades productivas y reproductivas.

En lo referente a las mujeres urbanas que trabajan en Rio-Tlan, cabe señalar el mantenimiento de la organización y/o ejecución del grueso de las 
actividades domésticas, se socialización y de cuidados en las mujeres; en este sentido, la participación de los hombres del grupo doméstico existe, pero en la mayoría de los casos es un 'apoyo' que se presta a la mujer. Se hace notar también la presencia de mujeres que esperan ser capaces de hacer del trabajo reproductivo no una 'ayuda' sino una corresponsabilidad de los futuros esposos o parejas. A pesar de no haberse examinado a fondo las políticas empresariales de las empresas de aventura de RioTlan, a simple vista no se identifican mecanismos tendientes a generar en un futuro medidas que promuevan la compatibilización de la vida laboral y familiar (entendida como una de las bases para un mayor involucramiento de los varones en el trabajo reproductivo de su grupo doméstico) y en cambio, sí destacan las largas jornadas de trabajo entre el personal cuando llega la temporada alta.

Contrario a lo que pudiera esperarse, la vida cotidiana de las mujeres no necesariamente transcurre en el barrio, en el caso de las mujeres urbanas que trabajan en el turismo de aventura en Rio-Tlan éstas además experimentan una parte de su cotidianidad alejadas físicamente de la casa y de los hijos; aparece una discontinuidad espacial, las mujeres recorren a diario entre 30 y $40 \mathrm{kms}$ desde un medio urbano a otro rural, pero las responsabilidades domésticas 'se quedan' en el medio urbano, por lo que deben generar diversas estrategias no sólo para hacer funcionar los acuerdos reproductivos de quienes permanecen en la ciudad sino también generar acuerdos para ellas mantenerse supervisando dichos acuerdos a pesar de la discontinuidad espacial. El cuidado de la casa y los hijos - principalmente esto último - mantiene la atención de las mujeres, centrada en el espacio reproductivo donde permanecen los hijos.

Con el fin de resolver unas responsabilidades que tradicionalmente se les han asignado, las mujeres recurren a diversas estrategias; en este sentido es posible identificar similitudes entre las medidas adoptadas por mujeres con hijos y sin hijos, de base las estrategias son las mismas: la cuota personal de trabajo reproductivo, la red familiar (especialmente la abuela), la contratación de servicios en el mercado (dependiendo las posibilidades económicas) o a través del Estado. De tal suerte que entre las mujeres sin hijos aparecen ya (de forma muy puntual) las que, con mucha probabilidad, serán las futuras estrategias a recurrir cuando nazcan los hijos.

La vinculación del trabajo doméstico a la identidad de las mujeres se mantiene, aunque no es inmutable, entre los grupos domésticos nucleares-conyugales; en ausencia de la madre es otra mujer quien continúa pendiente de buena parte de las actividades domésticas, de socialización y de cuidados. El permanecer fuera de la ciudad por más de 10 horas continuas todos los días requiere unas estrategias que consumen una cantidad de horas que no siempre es posible pueden contratar con el mercado o ser cubiertos por los servicios del Estado; por ello la figura de la abuela cuidadora se convierte en un elemento vital para la supervisión y/o realización del cuidado de los nietos.

A través de la revisión de las prácticas cotidianas se pretende también promover una sociedad más comprometida con la conciliación de la vida personal, familiar y comunitaria para las mujeres y los hombres; entendiendo el problema como uno social y no como un problema que debe ser enfrentado y resuelto exclusivamente por mujeres, entender lo anterior y trabajar en este sentido es una responsabilidad de toda la sociedad.

1 Se recomienda consultar a Giddens (1995); Wolf (2000); Lindón (Coord.) (2000); Chávez et al. (Eds) (2009).

2 Para fines de este artículo el trabajo reproductivo se subclasifica en trabajo doméstico, de socialización y de cuidados

3 El turismo de aventura en Rio-Tlan satisface de dos formas sus requerimientos de mano de obra, por lo general los puestos operativos y algunos de nivel medio se destinan para la población local y el resto, el denominado 'personal calificado' proviene de la capital del Estado o de otras ciudades también circunvecinas.

$4 \quad$ Si bien se persigue el objetivo de entrevistar a mujeres en diversos ciclos de vida, el rango de edad en cuestión representa los años cumplidos por la mujer más joven y la de mayor edad que componen la muestra de estudio y no ha sido fijado 'ex profeso'.

5 Cuando se cuestionó la razón de este proceder, las mujeres apelaron a los bajos salarios; sin embargo, en algunos casos aparece también la comodidad en la resolución de, principalmente, el trabajo doméstico, el ahorro que supone el vivir con los padres les permite el mantenimiento de cierto nivel de vida que independizándose no sería posible. En todo caso, asumen que con el matrimonio o el comienzo de una relación de pareja buscarán conformar nuevos grupos domésticos. 
6 Abreviatura cotidiana que se usa en los distintos países Latino Americanos para referirse a departamento.

$7 \quad$ P. ej., el mantenimiento de la casa y/o limpieza del automóvil familiar son tareas desempeñadas por los hombres, la cocina y la limpieza son tareas usualmente desempeñadas por las mujeres.

8 Abreviatura cotidiana que se usa en los distintos países Latino Americanos para referirse a refrigerador.

9 Con esto no se quiere decir que los hombres en grupos domésticos nucleares no realicen otras micro actividades de trabajo reproductivo, como suelen mantener las mismas micro actividades que realizan los hombres sin hijos se recomienda referirse al epígrafe anterior.

10 En este sentido llama la atención el uso que mantienen las abuelas con el mercado tradicional y el comercio de barrio al momento de realizar las compras cotidianas, mientras que se recurre a la hija para realizar compras en tiendas ubicadas en las grandes superficies donde se requiere un desplazamiento mayor que puede ser suplido a través del automóvil de la hija.

11 Dada la edad de las mujeres entrevistadas ninguna se ha visto en la necesidad de realizar servicios de cuidados intensivos destinados a sus padres, por eso la resolución de esta necesidad está ausente. No obstante no debe ser obviada pues es probable que en un futuro aun cuando los hijos se hayan hecho mayores ellas deban estar al pendiente de los padres y desarrollar otras estrategias para el cuidado de éstos, en una sociedad donde el servicio de cuidado de mayores proporcionado por el Estado no está tan extendido.

12 Inicialmente se había contemplado que entre los grupos domésticos con mayor poder adquisitivo y que contrataran trabajo reproductivo los hijos tendrían una baja participación en la realización de actividades de este tipo, independientemente del género; no obstante, también entre los grupos domésticos caracterizados por una situación económica menos solvente pero con mujeres de la familia los hijos mantenían una baja realización de este tipo de trabajo.

\section{Referências}

AYLLÓN, Maria Teresa. La territorialidad de la familia en la construcción de ciudadanía democrática". Ágora, v. 13, n. 1, p. 7 - 42, 2007.

BOSCO, Fernando; AITKEN, Stuart; HERMAN, Thomas. Women and children in a neighborhood advocacy group: engaging community and refashioning citizenship at the United States-Mexico border. Gender, Place and Culture, v. 18, n. 2, p. 155 - 178, 2011.

CHAMBERS, Kimberlee; HENSHALL-MOMSEN, Janet. From the kitchen and the field: Gender and maize diversity in the Bajío region of Mexico. Singapore Journal of Tropical Geography, v. 28, p. $39-56,2007$.

CHÁVEZ, Martha; GONZÁLEZ, Octavio; VENTURA, María (Eds). Geografía humana y ciencias sociales. Una relación reexaminada. Zamora: El Colegio de Michoacán, 2009.

HANSON, Susan. Spatial diversification and multipurpose travel: Implications for choice theory. Geographical Analysis, v. 12, p. 245 - 257, 1980.

HANSON, Susan. Gender and mobility: new approaches for informing sustainability. Gender, Place and Culture, v. 17, n. 1, p. 5 - 23, 2010.

HANSON, Susan; HANSON, Perry. The impact of married women's employment on household travel patterns. Transportation, v. 10, p. 165 - 183, 1981.

HANSON, Susan; HANSON, Perry. Gender and urban activity patterns in Uppsala, Sweden. Geographical Review, v. 70, p. 291 - 299. 1980.

GIDDENS, Anthony. Politics, sociology and social theory: Encounters with classical and contemporary social thought. Stanford: Stanford University Press, 1995.

JACKSON, Peter. ¿Nuevas geografías culturales?. Documents d'Anàlisi Geogràfica, v. 34, p. 41 - 51, 1999.

KATZ, Cindy; MONK, Janice (Eds), Full circles. Geographies of women over the life course. London: Routledge, 1993. 
LAEGRAN, Anne S. Domesticating home anchored work: Negotiating flexibility when bringing ICT based work home in rural communities. Geoforum, v. 39, p. 1991 - 1999, 2008.

LINDÓN, Alicia. Geografías de la vida cotidiana. En: HIERNAUX, Daniel; LINDÓN, Alicia (dirs). Tratado de Geografía Humana, Barcelona: Antrophos-UAM, 2006, p. 352 - 400 .

LINDÓN, Alicia. Trabajo, Espacios de Vida y Cotidianidad. La Periferia Oriental de La Ciudad de México, Scripta Nova, v. 6, n. 119, s/p. 2002.

LINDÓN, Alicia (Coord.). La vida cotidiana y su espacio-temporalidad. Barcelona: Ed. Anthropos, 2000.

LINDÓN, Alicia; HIERNAUX, Daniel. La Geografía Humana: un camino a recorrer. En: HIERNAUX, Daniel; LINDÓN, Alicia (Dirs). Tratado de Geografía Humana. Barcelona: Ed. Antrophos-UAM, 2006, p. 7 - 27.

MATTINGLY, Doreen; HANSEN, Ellen. Geographies of state power, protest, and women's political identity formation in Michoacán. Annals of the Association of American Geographers, v. 96, n. 2, p. 366 - 389, 2006.

McEVOY, Jamie; PERTZELKA, Peggy; RADEL, Claudia; SCHMOOK, Birgit. Gendered Mobility and Morality in a South-Eastern Mexican Community: Impacts of Male Labour Migration on the Women Left Behind. Mobilities, v. 7, n; 3, p. 369 - 388, 2012.

NELSON, Lise. Artesania, mobility and the crafting of indigenous identities among Purhépechan women in Mexico Journal of Latin American Geography, v. 5, n. 1, p. $51-77,2006$.

NELSON, Lise. Topographies of citizenship: Purhépechan Mexican women claiming political subjectivities. Gender, Place and Culture, v. 11, n. 2, p. $163-187,2004$.

QUINN, Bernadette. Care-givers, leisure and meanings of home: a case study of low income women in Dublin. Gender, Place and Culture, v. 17, n. 6, p. 759 - 774, 2010.

RADEL, Claudia; SCHMOOK, Birgit; McCANDLESS, Sussanah. Environment, transnational labor migration, and gender: Case studies from Southern Yucatán, Mexico and Vermont, USA. Population and Environment, v. 32, n. 2, p. 177 197, 2010.

RILEY, Mark. Bringing the 'invisible farmer' into sharper focus: gender relations and agricultural practices in the Peak District (UK). Gender, Place and Culture, v. 16, n. 6, p. 665 - 682, 2009.

ROSE, Gilian. Feminism and geography: the limits of geographical knowledge. Hong Kong: Blackwell Publishers, 1993.

SABATÉ, Ana; RODRÍGUEZ, Juana; DÍAZ, María. Mujeres, Espacio y Sociedad. Hacia una Geografía del Género. Madrid: Síntesis, 1995.

SCHWANEN, Tim; KWAN, Mei-Po; REN, Fang. How fixed is fixed? Gendered rigidity of space-time constraints and geographies of everyday activities. Geoforum, v. 39, p. 2109 - 2121, 2008.

TOWNSEND, Janet; AREVILLAGA, Úrsula; CANCINO, Socorro; PACHECO, Silvana; PÉREZ, Elia. Voces femeninas de las selvas, Texcoco: Colegio de Postgraduados; Centro de Estudios del Desarrollo Rural y Universidad de Durham, 1994.

POLLIO, Howard, HENLEY, Tracy; CRAIG, Thompson. The phenomenology of everydaylife.Empirical investigations of human experience. Cambridge: Cambridge University, 2006.

WOLF, Mauro. Sociologías de la Vida Cotidiana. Madrid: Cátedra Teorema, 2000.

Recebido em 16 de fevereiro de 2013. Aceito em 9 de abril de 2013. 